\title{
WOLFRAM DEMONSTRATIONS PROJECT PLATFORM AS A SUPPORT IN TEACHING
}

\author{
Izolda Gorgol ${ }^{1}$ \\ 1 Department of Applied Mathematics, Lublin University of Technology, Nadbystrzycka 38, 20-618 Lublin, \\ Poland, e-mail: i.gorgol@pollub.pl
}

Received: 2015.04 .07

Accepted: 2015.05.08

Published: 2015.06.01

\begin{abstract}
The article presents how Wolfram Demonstrations Project platform can support teaching of a great variety of subjects from very basic up to university level. The Wolfram Demonstrations Project is part of the family of free online services from Wolfram Research. General overview of the platform will be presented as well as a particular usage. The application concerns presenting to students certain numerical methods, namely one-step methods for Cauchy problem for ordinary differential equations.
\end{abstract}

Keywords: visualization, teaching.

\section{INTRODUCTION}

"A picture is worth a thousand words". This adage formulated at the beginning of 20th century [1] is now as up-to-date as never before. Nowadays, the young generation is brought up on pictures everywhere in everyday life. Therefore, visualization plays a great role in teaching, especially if some complicated notions and formulas are involved. Preparing such presentations requires some effort and time from the teacher. On the other hand, using the web resources one can bring together these two, apparently opposite, sides. Moreover it can encourage students to study on their own. Wolfram Demonstrations Project is one of the answers for such needs.

The Wolfram Demonstrations Project is part of the family of free online services from Wolfram Research created by Stephen Wolfram, a scientist and the author of MATHEMATICA, a system for modern technical computing. It started in 2007 and the idea was to bring computational exploration to the widest possible audience. It is an open-code resource that uses dynamic computation to illuminate concepts in science, technology, mathematics, art, finance, and a remarkable range of other fields. While writing this article there were 9926 interac- tive Demonstrations available. All of them are run for free on any standard Windows, Mac, or Linux computer. One does not even need MATHEMATICA. Any Demonstration can be interacted with using the free Wolfram CDF Player - for most platforms it can be used right in the web browser. One can also install CDF Player on the computer, download the Demonstration and use it offline. Those who have MATHEMATICA can also experiment and modify the code themselves [2].

\section{SHORT PRESENTATION OF WOLFRAM DEMONSTRATIONS PROJECT WEB PAGE}

The web page is available at the URL address http://demonstrations.wolfram.com/. Entering the page one have the access to all Demonstrations. Some of them are featured, and after pointing them the description is visible. As an example "Spiral Formations from Iterated Exponentiation" is presented in Figure 1.

Just above featured demonstrations on righthand side there is a link to the new and updated demonstrations (Figs. 2 and 3 ).

At the bottom of the homepage one has the possibility to browse Demonstrations by topic. 


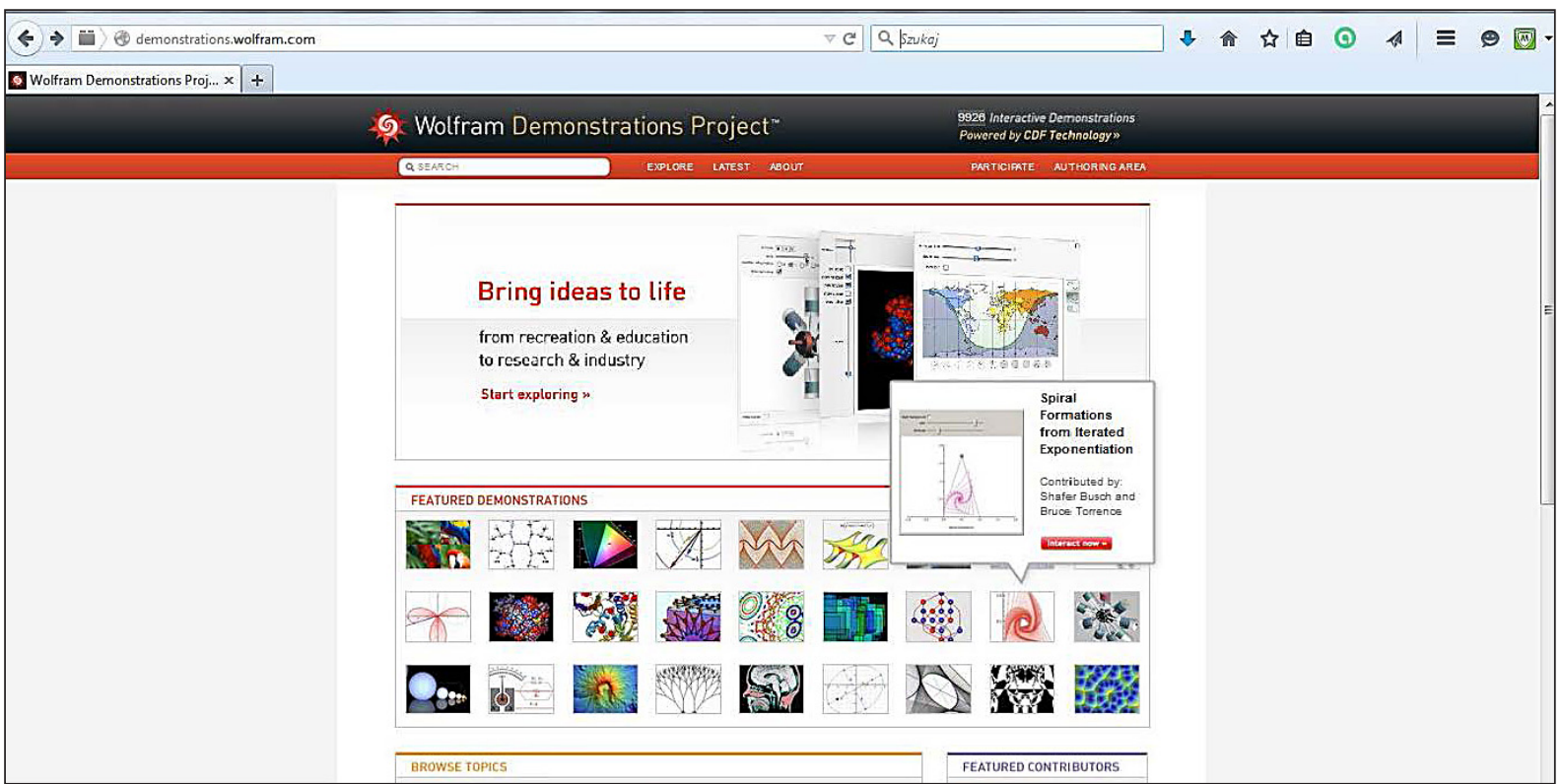

Fig. 1. Wolfram Demonstrations Project homepage (top part)

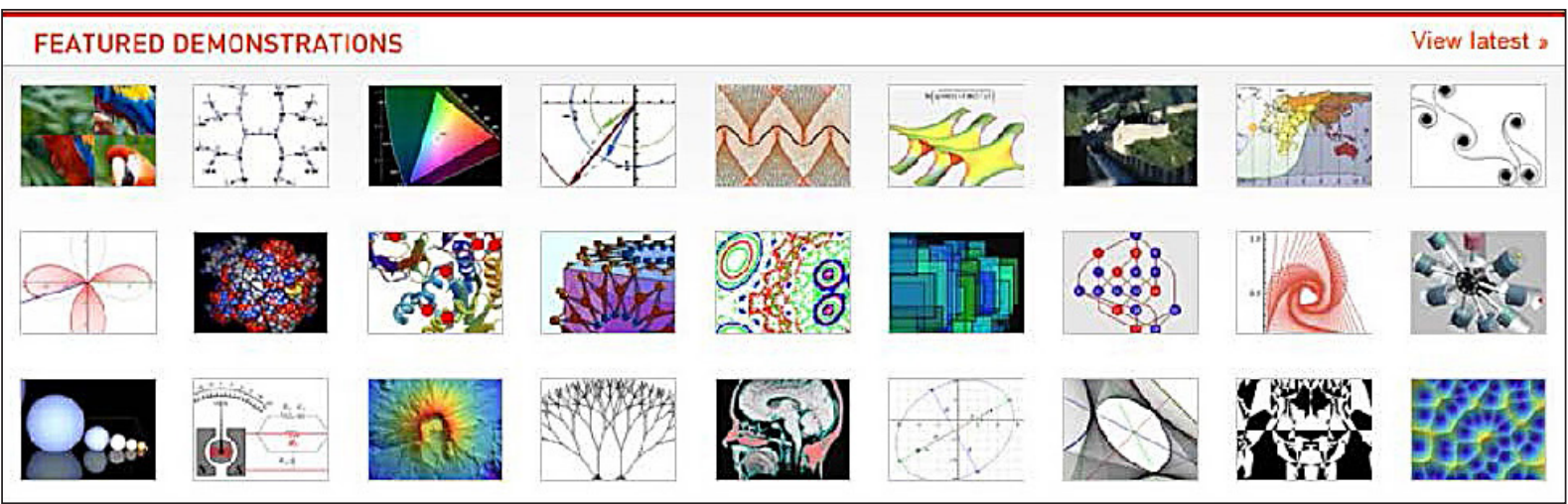

Fig. 2. View latest Demonstrations

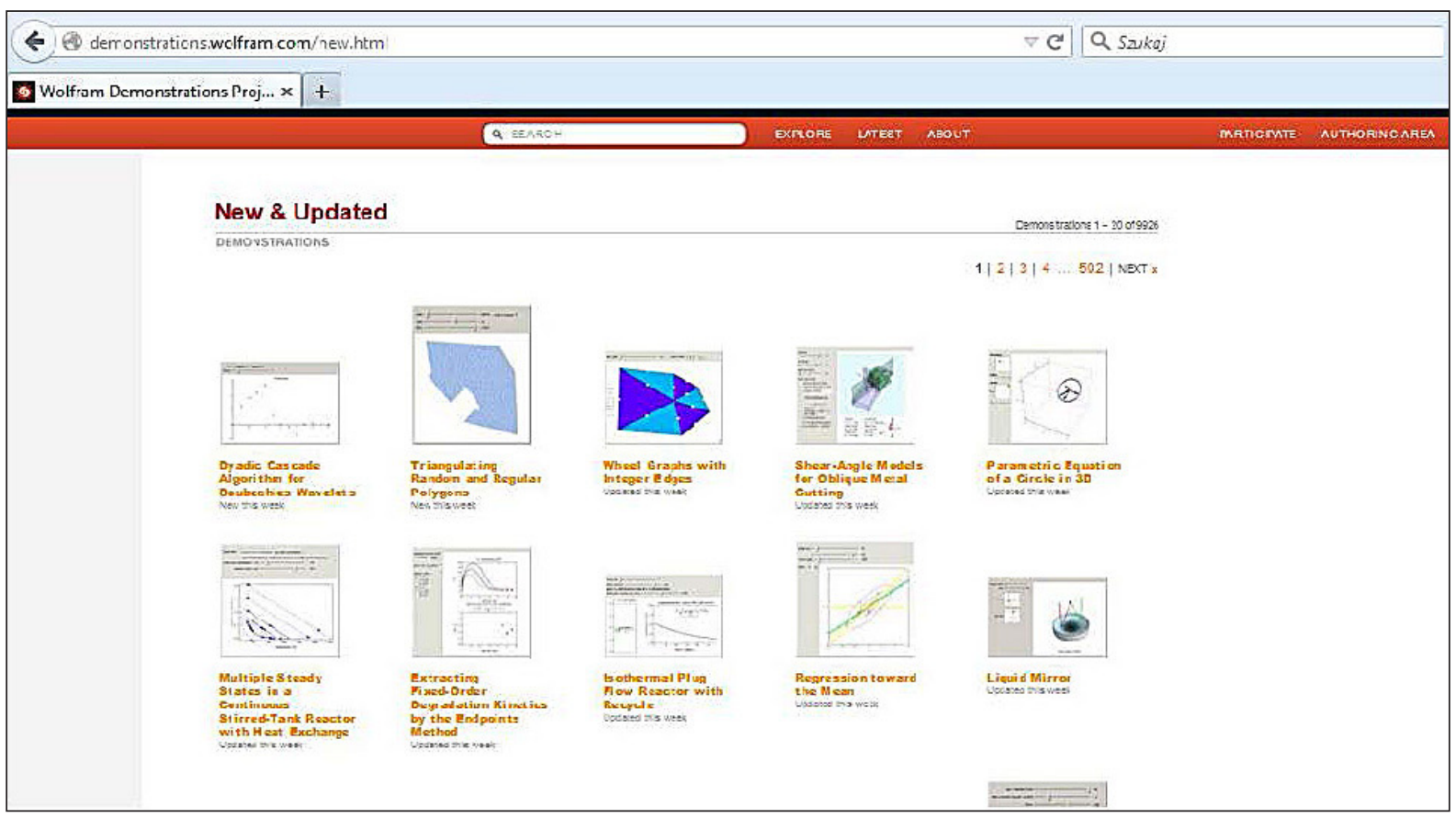

Fig. 3. New and updated Demonstrations 
The main topics are:

- Mathematics,

- Computation,

- Physical Sciences,

- Life Sciences,

- Business and Social Systems,

- Systems, Models and Methods,

- Engineering and Technology,
- Our World,

- Creative Arts,

- Kids and Fun,

- MATHEMATICA Functionality.

Apart from that featured contributors are presented. At the very bottom of the page there are links to other services from Wolfram Research (see Fig. 4).

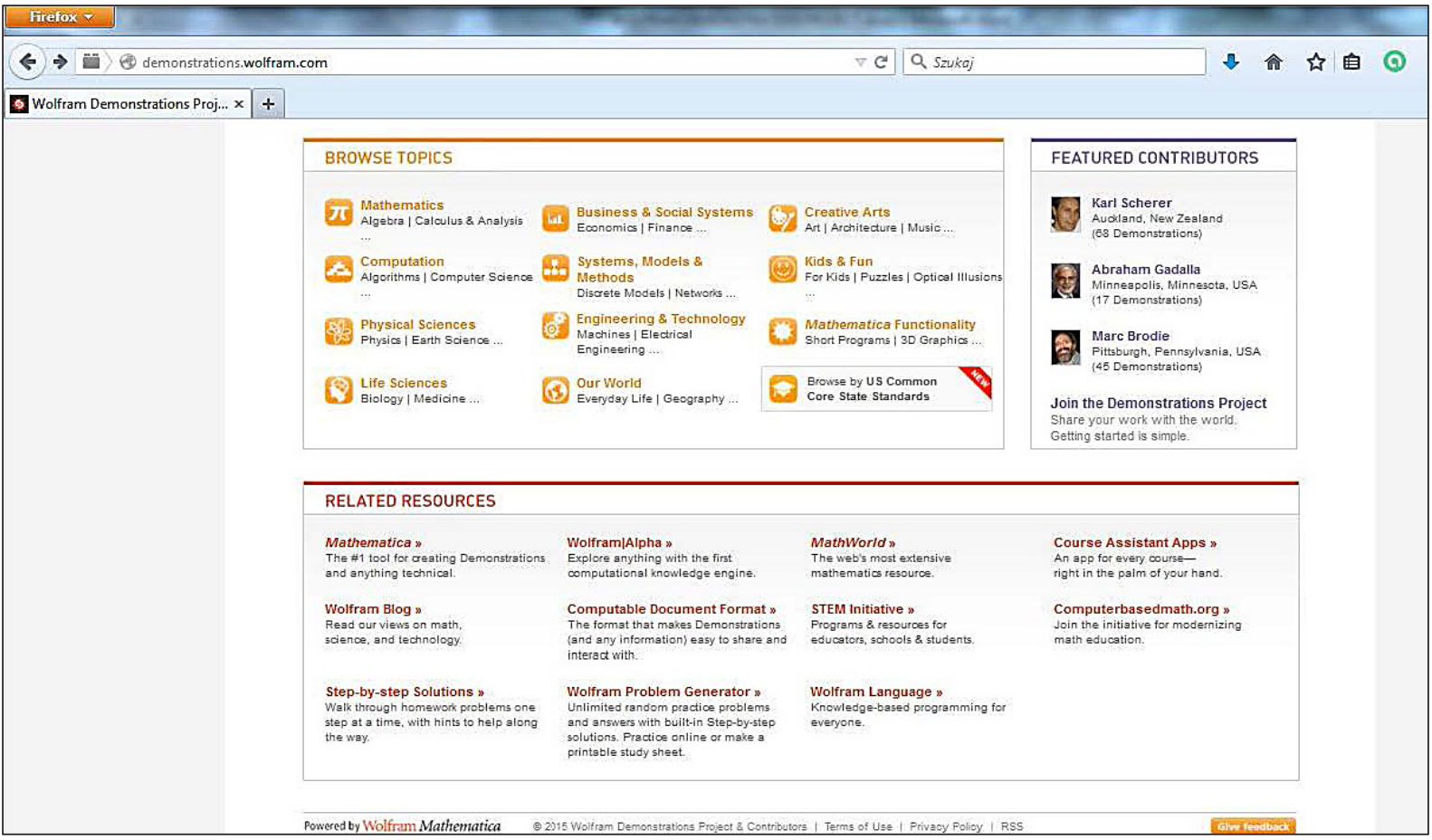

Fig. 4. Wolfram Demonstrations Project homepage (bottom part)

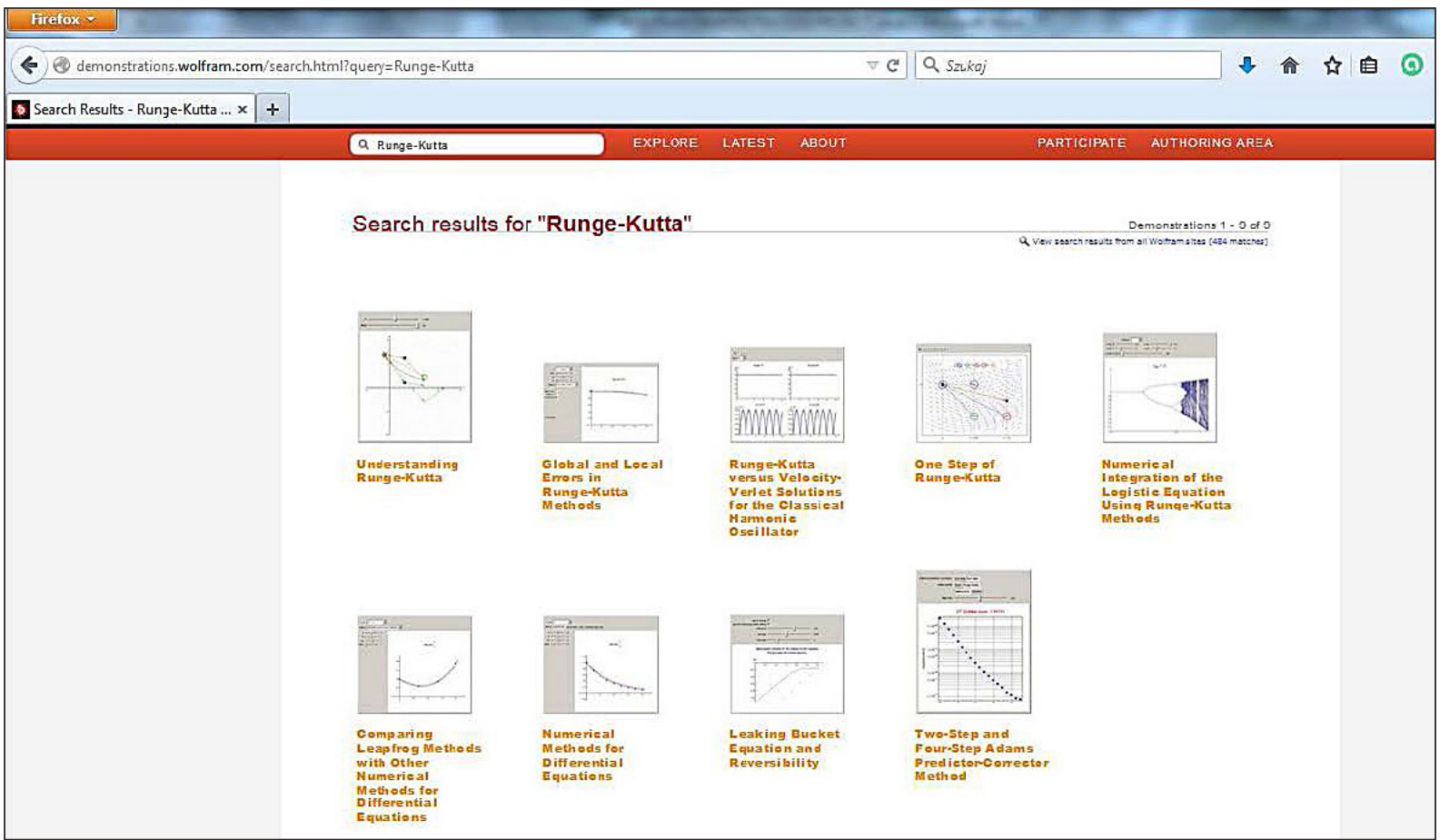

Fig. 5. Demonstrations available for "Runge-Kutta" 
On the top of main page and each subpage there is a toolbar which allows to browse Demonstrations according to a chosen expression. In Figure 5 there is an example for Demonstrations available for "Runge-Kutta" question.

\section{EXAMPLE OF USAGE}

One of the topics which are presented to students are the numerical methods for solving dif- ferential equations. Let us consider the example of a Cauchy problem for ordinary differential equations, i.e. determining the function $y=y(x)$, $a \leq x \leq b$, such that $y^{\prime}=f(x, y), y(a)=y_{0}$. It is known that this problem has an unique solution while the function $f$ fulfills specified assumptions. There is a variety of numerical methods which can be used for solving this problem. Among them there are such one-step methods as explicit and implicit Euler's methods of order 1, modi-
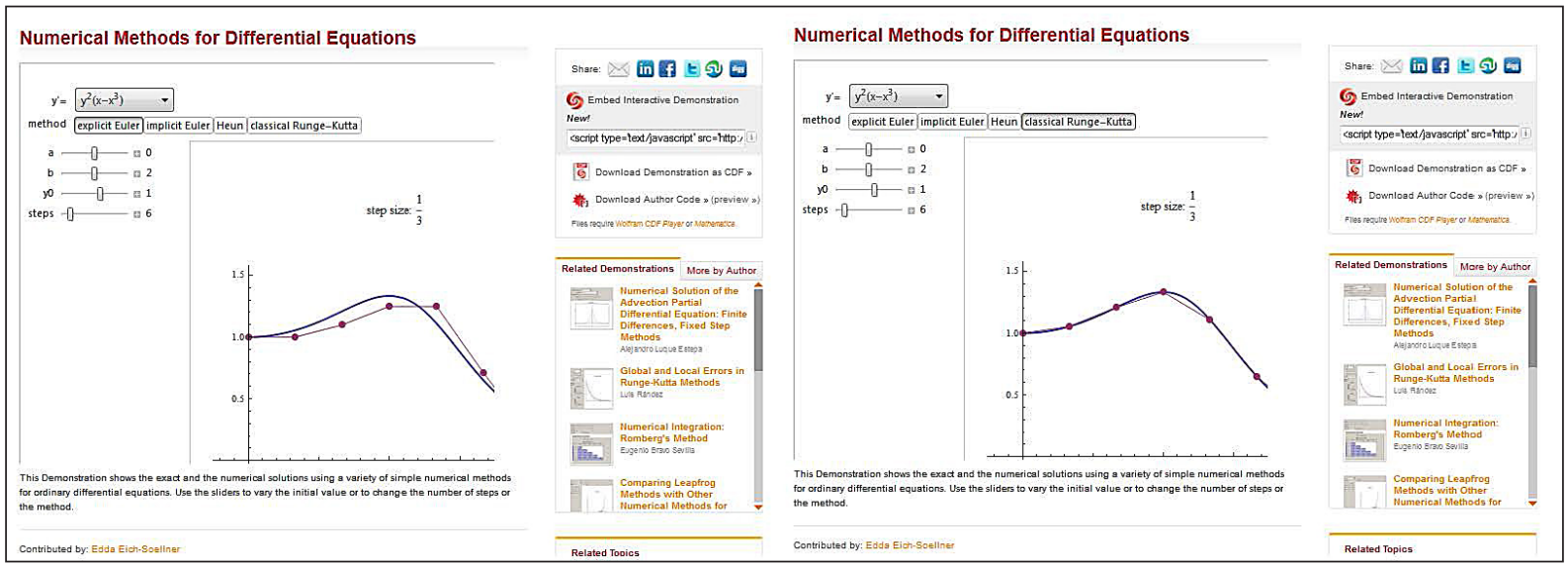

Fig. 6. Comparison of explicit Euler and classical Runge-Kutta

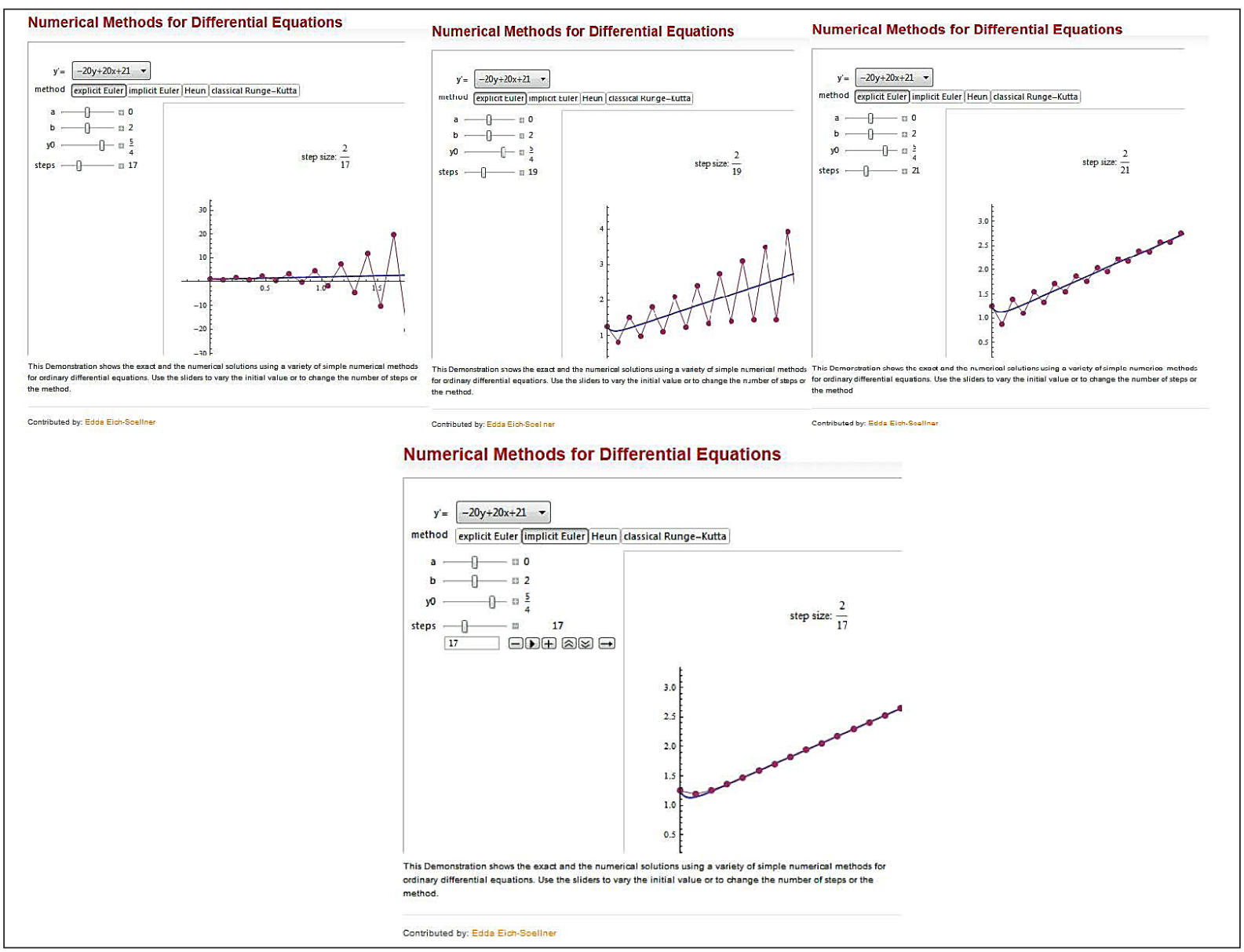

Fig. 7. Explicit versus implicit Euler's method 
fied Euler's or Heun's method of order 2 or Runge-Kutta methods of different orders. They have various accuracy and complexity (cf. eg. [3]). It is very useful that apart from presenting formulas one can compare these methods by visualizing the solutions obtained by different methods. Using the demonstration "Numerical Methods for Differential Equations" contributed by Edda Eich-Soellner it is possible to compare explicit and implicit Euler's methods, Heun's method and classical RungeKutta method of order 4 . There are seven functions $f$ that can be chosen. For each of them an exact solution is shown and it is possible to change dynamically the initial condition, a range $<a, b>$ and the number of steps. It allows to show the students how the methods work in a very easy way.

In Figure 6 it is presented the comparison of numerical solutions with explicit Euler and classical Runge-Kutta methods of the initial Cauchy problem $y^{\prime}=y^{2}\left(x^{3}-x\right), y(0)=1$ on the interval $<0,2>$ with 6 steps. A blue line denotes the exact solution and the purple dots are values obtained by particular methods.

Moreover, it is very easy to ilustrate the behaviour of explicit and implicit methods on a stiff equation. In Figure 7 it is presented the comparision of explicit Euler's methods for the problem $y^{\prime}=-20 y+20 x+21, y(0)=5 / 4$ on the interval $<0,2>$ with 17,19 and 21 steps with implicit one with 17 steps. In that way it is very easy to confirm the theoretical comparison of these methods, explain why the step size is so important in explicit method while implicit one gives good results with relatively long step.

Moreover, each subpage provides the link to basic theory connected with the demonstration as well as to related demonstrations, for instance to "Global and Local Errors in Runge-Kutta Methods" as related to presented demonstration. Additionally, other demonstrations by the same author can be found.

\section{CONCLUSIONS}

The benefits of introduction dynamic mathematics demonstrations into the teaching and learning process are presented. The aim of each teacher is to be as clear as possible. It is shown that Wolfram Demonstrations Project platform allows to enrich the lecture to achieve this aim with very little effort. For instance, it is hard to imagine teaching numerical methods without computer tests nowadays. That is why the example from this branch of knowledge was chosen. It occurs that even complete lack of programming skills is not an impediment for the teachers to confirm the theory presented during the lecture by a computer presentation.

\section{REFERENCES}

1. „Wikipedia,” [Online]. Available: http:// en.wikipedia.org/wiki/A_picture_is_worth_a_ thousand_words. [Accessed 9.03.2015].

2. Wolfram Research, „Wolfram Demonstration Project," Wolfram Research, [Online]. Available: http://demonstrations.wolfram.com/about.html. [Accessed 9.03.2015].

3. Cheney W., Kincaid D., Numerical mathematics and computing, Belmont: Thomson Brooks/Cole, 2008. 